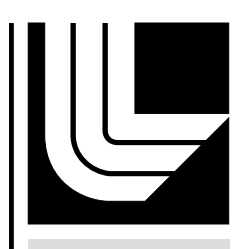

LAWRENCE LIVERM ORE N A TIO N A L LABORATORY

\title{
Correlation Between Degradation and Broadness of the Transition in $\mathrm{CICC}$
}

N. Martovetsky

March 7, 2013

7th Workshop of Mechanical-Electromagnetic Properties of Superconducting Materials

Aix-enProvence, France

March 12, 2013 through March 14, 2013 
This document was prepared as an account of work sponsored by an agency of the United States government. Neither the United States government nor Lawrence Livermore National Security, LLC, nor any of their employees makes any warranty, expressed or implied, or assumes any legal liability or responsibility for the accuracy, completeness, or usefulness of any information, apparatus, product, or process disclosed, or represents that its use would not infringe privately owned rights. Reference herein to any specific commercial product, process, or service by trade name, trademark, manufacturer, or otherwise does not necessarily constitute or imply its endorsement, recommendation, or favoring by the United States government or Lawrence Livermore National Security, LLC. The views and opinions of authors expressed herein do not necessarily state or reflect those of the United States government or Lawrence Livermore National Security, LLC, and shall not be used for advertising or product endorsement purposes. 


\section{Correlation between degradation and broadness of the transition in CICC}

Nicolai Martovetsky

LLNL/ORNL US ITER, 1055 Commerce Park Dr. ORNL, Oak Ridge, Tennessee, 37831

\section{ABSTRACT}

Cable in Conduit Conductor (CICC) performance is characterized in terms of relationships involving the electric field $(\mathrm{E})$, voltage $(\mathrm{V})$, temperature $(\mathrm{T})$, current $(\mathrm{I})$, magnetic field (B), and strain. Development of the electrical field in the V-T or V-I transitions in CICC is exponential, and in the coordinates $\log \mathrm{E}$ vs $\mathrm{T}$ or $\log \mathrm{E}$ vs I looks as a straight line.

ITER Nb3Sn CICCs show degradation of properties versus load cycles that could be attributed to plastic deformation of the Nb3Sn strands or fracture of the superconducting filaments. The degradation is expressed in terms of reduction of the current sharing temperature Tcs or critical current Ic, respectively. It was noticed long ago that degradation is accompanied by a significant broadening of the V-T or V-I transition, that looks like a change in the slope in the semi-log coordinate plot.

This paper presents some systematic observations of correlations between the critical parameters and broadness of the transition in many conductors tested in CICC. In most cases, the broadness of the transition seems to be a more sensitive indicator of the conductor damage even in cases when Tcs degradation is not clearly seen. Tcs degradation typically becomes obvious later in the cycling, especially after warm up and following cooldown and more cycling.

In some cases, a CICC manifests temporary or even a permanent growth of Tcs with load cycles, especially in the latest measurements of the CS conductors with short twist pitches. A possible mechanism of degradation that allows qualitative explanation of this phenomenon is discussed, which is supported by the voltage measurements on the cable in TFUS1 with the voltage taps penetrating the jacket to the cable.

\section{Introduction}

Since middle of 1990's, when an intensive development of the Nb3Sn CICC took place, a wealth of data on degradation of the high current Nb3Sn CICC has been developed. However, this knowledge has not yet produced a quantitative correlation that would allow predicting performance of the CICC from the properties of the strand, cabling pattern, void fraction and jacket properties and geometry.

Performance of the CICC for ITER TF observed for the period of 2007-2010 in the SULTAN facility, where essentially identical cables and jackets were used by different suppliers, showed no correlation between performance of the strands and performance of the CICC [1-2]. More recent data of ITER TF conductors tested in the SULTAN facility in 2011-2012 started showing a reasonable correlation between the CICC and the strands performance before cycling and degradation associated with it [3]. 
There is no question that the production of the ITER CICC became more mature than in the development phase; however it is not clear why the CICC performance was less predictable from the strand performance before.

Degradation of the CICC with cycles remains difficult to predict. The load cycles are result of the repeated IxB electromagnetic forces pushing the cable every charge. The design life of ITER central solenoid (CS) is 60000 load cycles. From the practical stand point it is sufficient to find a configuration of the strand-cable-jacket void fraction where degradation does not exist, but this is not an easy task. Until recently, such a combination of parameters was not established for the CS conductor and still is not established for the TF conductors. Recently, the so-called short twist pitches introduced by D. Bessette into CS cable design showed excellent results in the SULTAN tests for five conductors with different strands [4-6]. This phenomenon is still not well understood quantitatively and did not improve our predictive capability. However, demonstrating the existence of a cabling pattern that solves the degradation problem is very significant for ITER and for CICC development.

In this paper we try to correlate the evolution of the conductors that experienced some degradation with the conductors that did not show a significant degradation in order to see how broadness of the transition correlates with the degradation, which is expressed in terms of decreasing Tcs versus cycles and thermal warm-up and cool-downs.

It is natural to expect that if the degradation in the CICC takes place because of some damage in the strands (like fracture of filaments or plastic deformation due to bending and pinching of the strands) we should see an increase of broadening of the transition to the normal state. If we observe broadening of the transition and simultaneously degradation of the Tcs that means that the degradation is caused by the damage in the strands.

Sometimes Tcs changes (usually to a lower value or sometimes higher) but broadening of the transition is not observed. This may mean that that EM forces brought the strands to more favorable conditions than it was before the cycling, but after several hundred of cycles, a saturation takes place and fracture of filaments ends, if it occurred at all.

\section{Description of the electrical growth in superconductors with varying temperature, current and magnetic field}

It is a well-known fact that the development of the electrical field in practical high current density superconductors is not abrupt, but exponential [7] versus current, magnetic field or temperature. That means that for some applications, the superconducting transition cannot be described by a single "critical” parameter. A minimum two parameters are needed for a transition description, one is a "conventional" critical parameter and another one is a broadness of the transition.

The original suggestion of the exponential growth of the electrical field in hard superconductors can be traced to the theory of thermal activation of the flux vortices [8], but the detailed studies of the practical superconductors revealed a significantly more complicated relationship of the electrical field development. Since then there were many attempts to explain the exponential growth by inhomogeneity of the grain properties. A normal distribution of the Tc 
or jc in the domains, like grains, resulted in exponential growth of the electrical field versus temperature. A longitudinal inhomogeneity, also known as "sausaging" of the filaments, results in a little more complicated shape of the electrical field development.

With a good approximation, most of the strands and CICC at reasonably high current densities describe the electrical field development of practical superconductors as follows [7]:

$$
E=E_{c} \exp \left(\frac{T-T_{c s}}{T_{o}}+\frac{I-I_{c}}{I_{o}}+\frac{B-B_{c s}}{B_{o}}\right)
$$

This relationship shows that the electrical field growth is exponential versus field, current and magnetic field. The parameters that describe the broadness of transition, the increments: To, Io and Bo are not constants, but relatively weak functions of the magnetic field and temperature and do not depend on transport current, but do depend on the critical current.

This relationship (1) is observed on the majority of the SULTAN samples measured in the ITER qualification effort from all six countries-suppliers of the ITER conductors; the strands for which were provided by 9 strand suppliers.

In the SULTAN tests the cleanest transition is obtained for the V-T curves measured at a constant current. This is due to the fact, that in large CICC conductors a voltage-current, V-I transition generates an inductive noise, changes the magnetic field, may introduce current redistribution in the joints and most importantly, generates an additional temperature rise in the joints and due to self-heating in the area that generates the electrical field. Therefore V-I characteristics, where many parameters are changing, are not as clean as the V-T transitions.

In this case the relationship (1) becomes as follows:

$$
E=E_{c} \exp \left(\frac{T-T_{c s}}{T_{o}}\right)
$$

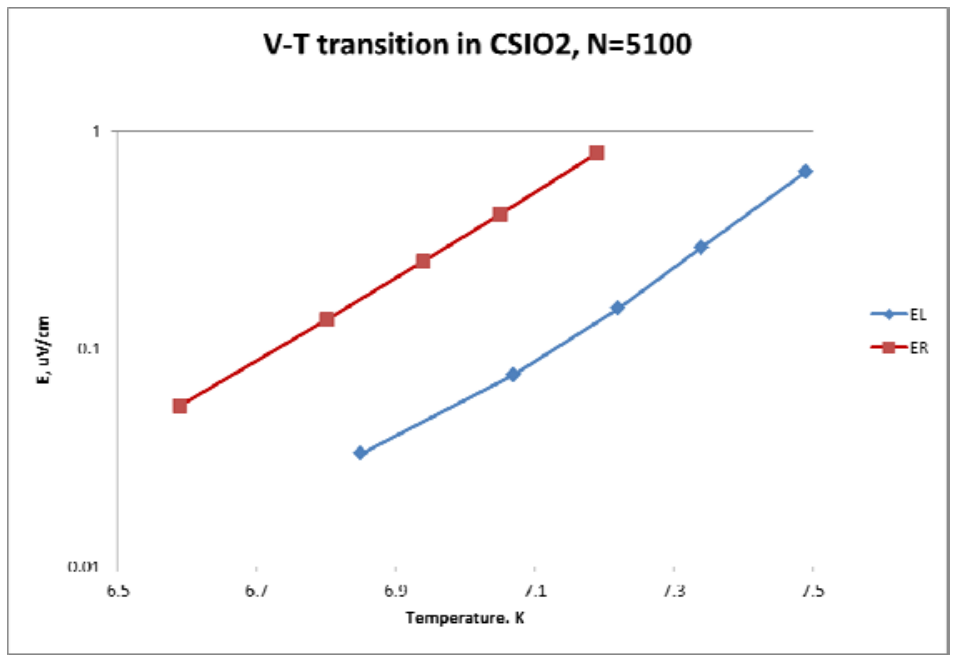


Fig. 1. A typical V-T transition of a CS conductor CSIO2 from SULTAN test [10] in semi-log coordinates. The EL and ER refer to the left and right legs of the SULTAN sample (courtesy of $B$. Stepanov, CRPP).

Fig. 1 presents a typical example of the processed V-T characteristic, which is taken from the ITER CICC in the SULTAN testing.

In order to see the effectiveness of the CICC and its utilization of the strand properties, it is necessary to compare the CICC V-T characteristic with the strand V-T characteristic.Unfortunately, the direct comparison is not straight forward. Most of the strands are measured in the V-I type of measurements. On the strand level, the overheating is low, the current is low and therefore there is a low effect of the magnetic self-field, so only Io parameter (or Nvalue) is measured. In the ITER and HEP community, a popular representation of the V-I characteristic is:

$$
E=E_{c}\left(\frac{I}{I_{c}}\right)^{N}
$$

where the Ic and $\mathrm{N}$ are usually reported from the tests.

This correlation is almost indistinguishable from the exponential transition in a wide range of electrical field change if $\mathrm{N}$ is high (say $\mathrm{N}>20$ ). This is illustrated in Fig. 2 for a range of Io/Ic values of 0.05 to 0.16 and $\mathrm{N}=20$ to 6

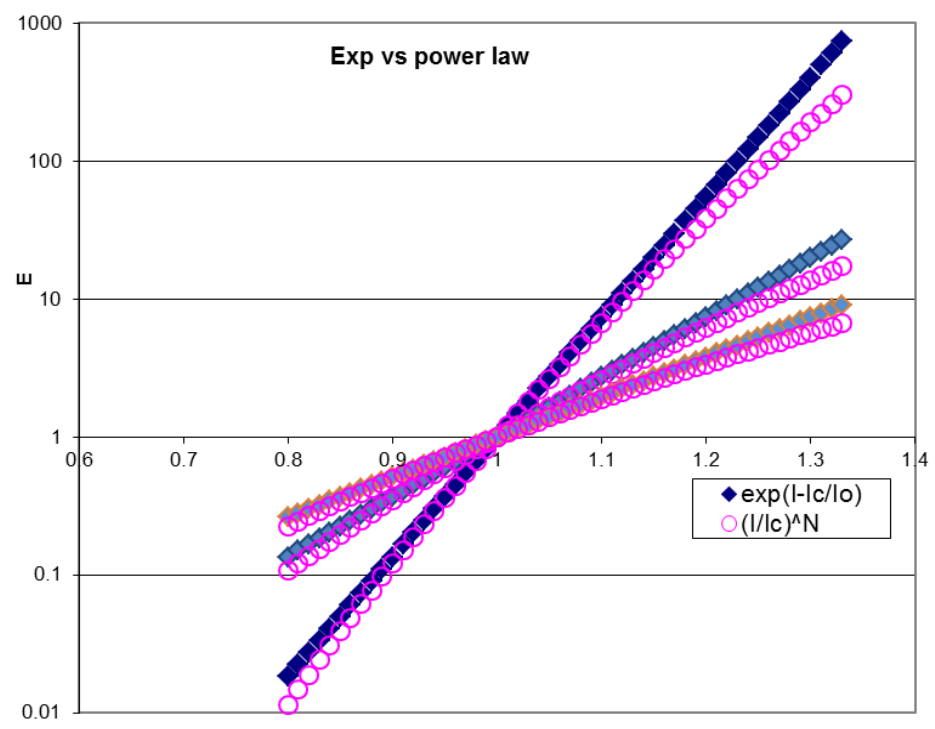

Fig.2. Comparison of the exponential and power functions with $I c / I 0=N=20,10$ and 6

The correlation (3) is a purely empirical and historically accepted by some researchers, and the $\mathrm{N}$-value is often quoted in the specification of the strands. The correlation between the Io parameter and $\mathrm{N}$-value to match the slope at the point of the Ic definition becomes as follows: 


$$
I_{o}=\frac{I_{c}}{N}
$$

Extraction of the $\mathrm{N}$ value is not very straight forward for $\mathrm{V}-\mathrm{T}$ characteristics processing. On the other hand, measurements of the V-T characteristics for strands are much more difficult and less common, than for the CICC. Thus, we need some convergence rules from V-I to V-T.

Taking partial derivatives of the electrical field versus temperature and current, one can easily find by differentiating (1) that:

$$
I_{o}=\left(\frac{\partial I_{c}}{\partial T}\right)_{E} T_{o}
$$

For the SULTAN sample, the derivative is routinely measured in the SULTAN test campaign, the Tcs at lower currents is measured, from which the derivative can be easily determined. For the strands, this correlation is also mandatory and therefore it is available through the reported correlation of Ic ( $T$, B, eps).

Combining (4) and (5) we can express To through $\mathrm{N}$ in the following way:

$$
T_{o}=\frac{1}{\left(\frac{\partial I_{c}}{\partial T}\right)_{E} N}
$$

In general, the $\mathrm{N}$-value as a measure of stability and broadness of transition is sometimes misleading. The measure of stability in superconductors is the level of electrical field of take off; the higher the electrical field, the higher the stability [11]. The take-off field can be expressed as:

$$
E_{t o}=\frac{h P T_{o}}{I_{c}}
$$

For a similar class of conductors, like $\mathrm{Nb}_{3} \mathrm{Sn}$, the $\mathrm{N}$ value is unambiguously connected to $\mathrm{T}_{\mathrm{o}}$ as one can see in formula (6). But if we compare different conductors and using $\mathrm{N}$-value as a figure of merit, we can discover that the same $\mathrm{N}$ value, say 20 in NbTi conductors and in Nb3Sn conductors, will have significantly different parameters of the To. For Nb3Sn strands the To typically are $0.01-0.02 \mathrm{~K}$ and for Nb3Sn the To is typically 0.2-0.5 K. For the NbTi strands and CICC, the To is an order of magnitude lower. This is the exact reason why NbTi CICC cannot be measured at $10 \mu \mathrm{V} / \mathrm{m}$ at high currents; the take off takes place often even before it can be observed. If a stable voltage is not observed, it cannot be determined if the current reached the critical value or if it is a premature quench and the true CICC performance is masked by uncontrollable level of the noise. 


\section{Observation of the broadness of transition correlation with degradation in CICC}

One of the first systematic observations of the correlation between performance of the Nb3Sn conductors and degradation was the model coil effort on T-15 tokamak [12]. The T-15 monolithic conductor was made by an electroplating deposition of copper on a Rutherford type cable with two copper tubes. The T-15 was done by a react and wind method.

It was observed on the straight samples, and model coils with different radii of curvature that the parameter of Ic*B ratio changed from $85 \mathrm{kAT}$ to $45-50 \mathrm{kAT}$ as the bend radius becomes smaller and smaller. It was observed that the $\mathrm{N}$-value decreased during this transformation by a factor of 5 , from 20-30 to 5 in the mostly degraded cases.

The noticeable observation was that the broadness of transition to normal state is more sensitive indication of degradation than the degradation itself, measured by Tcs or Ic. Later these observations were checked on many magnets and conductors. In most cases, they were confirmed.

Before discussing degradation and broadness of the transition correlations, we need to see how to compare the CICC with the strand properties that the CICC is made of. Very often people comparing performance of the strand with performance of the CICC miss the point that the CICC is in a different state of strain, but more important, the strands operate at a different current.

One of the first correlations of the $\mathrm{N}$-value with the critical current was reported in Ref. [13]. It was showed that the broadness of correlation (N-value) was correlated to the critical current Ic of the strand. That was confirmed by study of practically all strands characterized for the CSMC and TFMC programs. Quickly after the distribution of this study [13], it became a widely accepted correlation.

Fig. 3. Shows results of the Furukawa $\mathrm{N}$ vs Ic for strand characterization based on data from [14]. It shows, that the $\mathrm{N}$ vs Ic can be approximated by a function of

$$
N=A I_{c}^{b}
$$

where the " $\mathrm{b}$ " is typically in the range of 0.3 to 0.6 . 


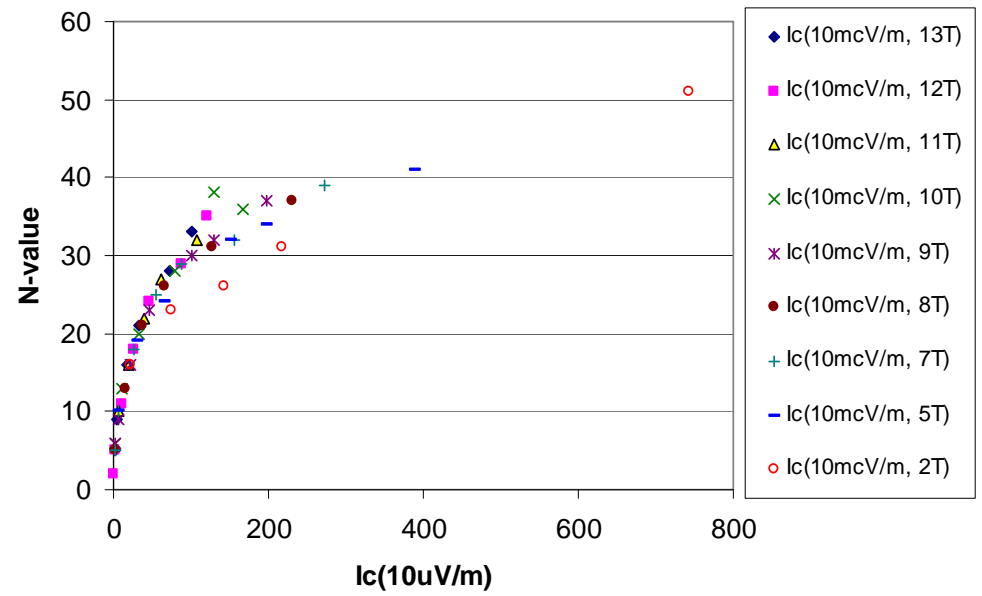

Fig. 3. Correlation between the broadness of the $\mathrm{V}-\mathrm{T}$ transition ( $\mathrm{N}$-value) and critical current at the constant strain, Furukawa strand, data from [14].

It was also noticed [13] that the strain changes the N(Ic) correlation significantly (see Fig. 4.)

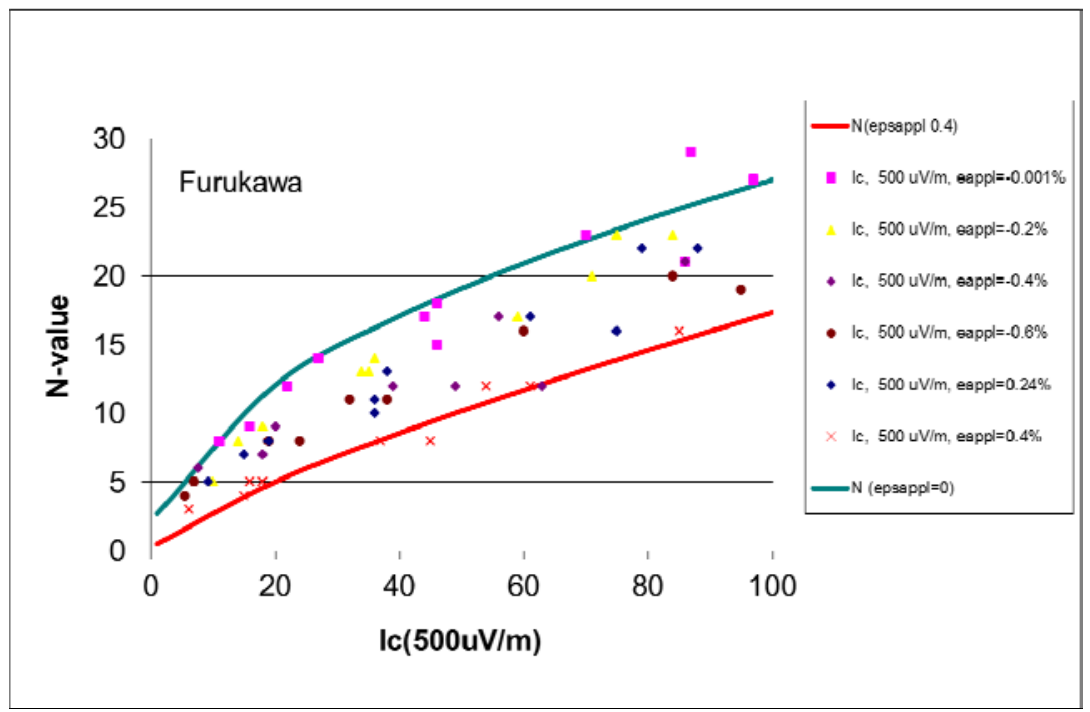

Fig. 4. $\mathbf{N}$ value vs Ic correlation for selected data for Furukawa strand in the wide range of the strain (from $-0.6 \%$ to $0.4 \%)$, temperature (4-14 K) and field (5-13 T).

The solid lines in Fig. 4 are approximation of the N(Ic) for zero and $0.4 \%$ tensile strains. As one can see, the tensile or compressive strain, both increase the width of transition (reduce Nvalue), even within the reversible region. The tensile strain, however, suppresses the the $\mathrm{N}$-value stronger (raw data were taken from [14]). The tensile strain of $0.4 \%$ reduces the $\mathrm{N}$-value by a factor of 2 from the strain free conditions.

Thus, comparison of the N-value of the strand and CICC must take into account that the strand in CICC usually operates at a significantly lower current per strand and in a compressive strain conditions. 
In the CS Insert, the operating current was 34.7 A per strand, where the N-value is about 12 in a free strain strand (Fig. 3), while at $120 \mathrm{~A}$, a typical value of Ic in $12 \mathrm{~T}$ on an ITER barrel, the $\mathrm{N}$-value is about 30 . The measured $\mathrm{N}$-value in the CS Insert was near 8.5 before cycling and 7.4 after the 1000 cycles [15]. Thus, it is not fair to compare the $\mathrm{N}$-value from the strand tests with the N-value full scale conductors as in in the coils or SULTAN tests. Evolution of the Nvalue in the CSInsert before load cycles and after 1000 cycles is shown in Fig. 5.

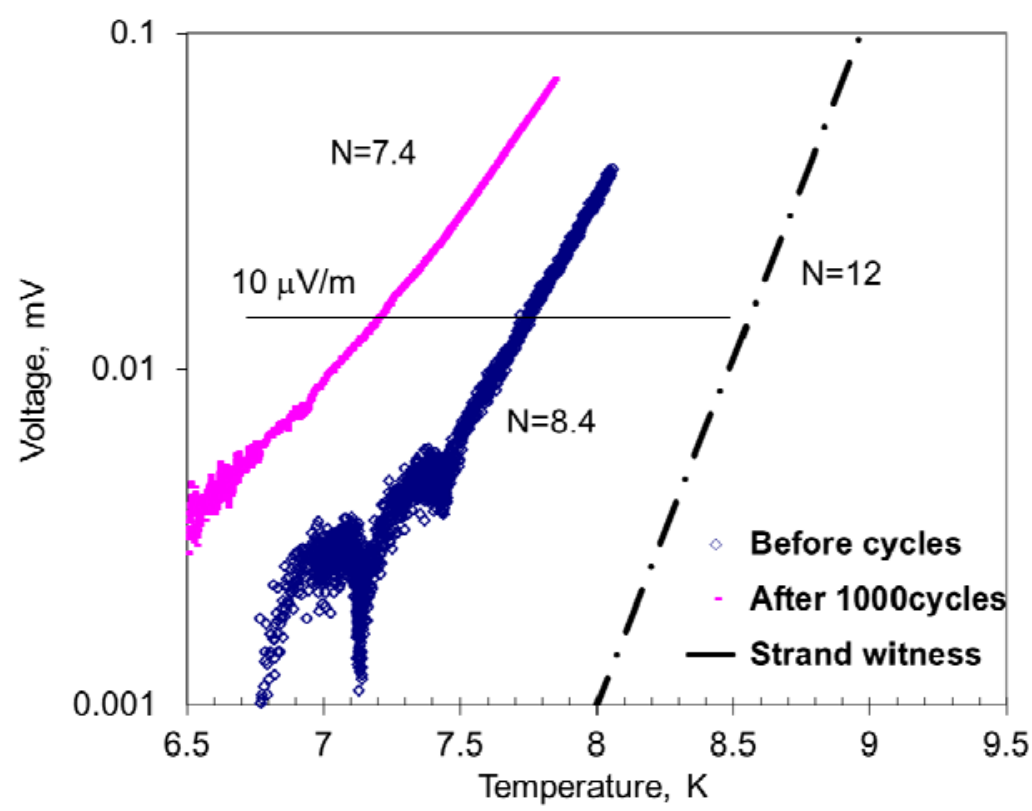

Fig. 5. Semi-log plot of the V-T traces of the Furukawa strand and CS Insert before cycles and after 1000 cycles.

From Fig. 5 we can see that degradation of the CSI performance corresponds to a reduction of the $\mathrm{N}$-value from 12 to 7.4 or increase of the To parameter from $0.2 \mathrm{~K}$ in the strand to $0.42 \mathrm{~K}$ after 1000 cycles. The following testing did not show further drop of the $\mathrm{N}$-value that lead to the conclusion that degradation with cycles ended.

Let us now see how recent TF and CS conductors behaved during cyclic testing in SULTAN facility. Fig. 6 shows a typical example of a conductor where the degradation is apparent, but relatively slow. However, the broadness of the V-T transition is significantly more pronounced that leaves no doubt that the degradation is associated with a fractured filaments or depressing strain conditions, like plastic pinching of the wires at the cross overs. Parameter To almost tripled after 17000 cycles, while Tcs dropped by about $1 \mathrm{~K}$. 
Correlation between degradation and broadness of the transition in CICC

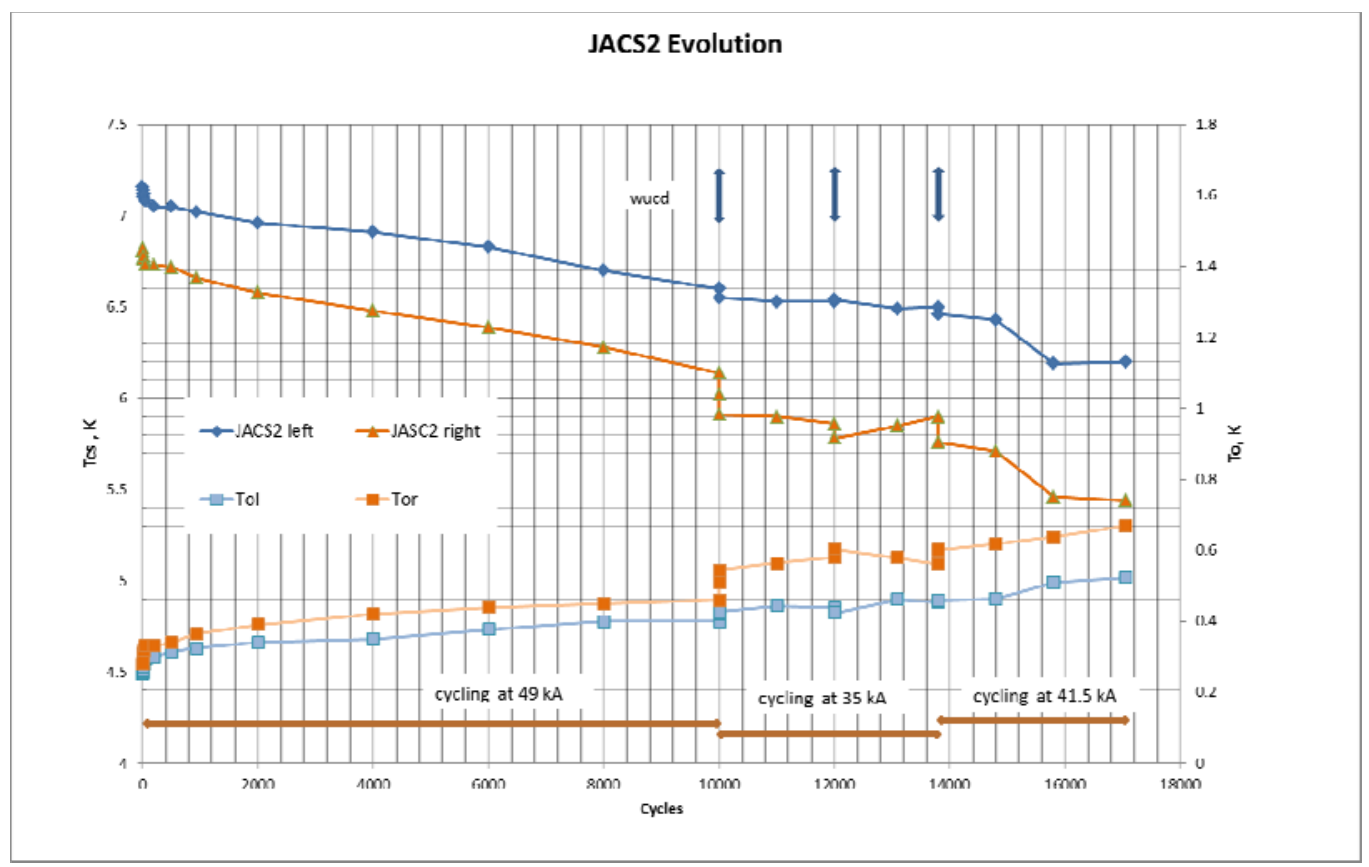

Fig. 6. A typical degradation of the CICC as a result of load cycles in the SULTAN facility in CSJA2 conductor that went through a complex history of load.

It is noticeable that when the Tcs is apparently stabilizing at $35 \mathrm{kA}$ cycling and To is still going upwards, the degradation is still in progress.

There were a few conductors where Tcs saturation versus cycles was observed, like TFUS1 [16] after several hundred cycles. The most impressive is the saturation in the CS conductors with the short twist pitches [4-6].

Fig. 7 shows correlation between the Tcs and To in the CSJA3 conductor. The Tcs even experiences a slight growth, but the To saturates after a slight increase. The two processes going hand in hand give a good confidence that the damage in the CICC is not taking place with cycles. 


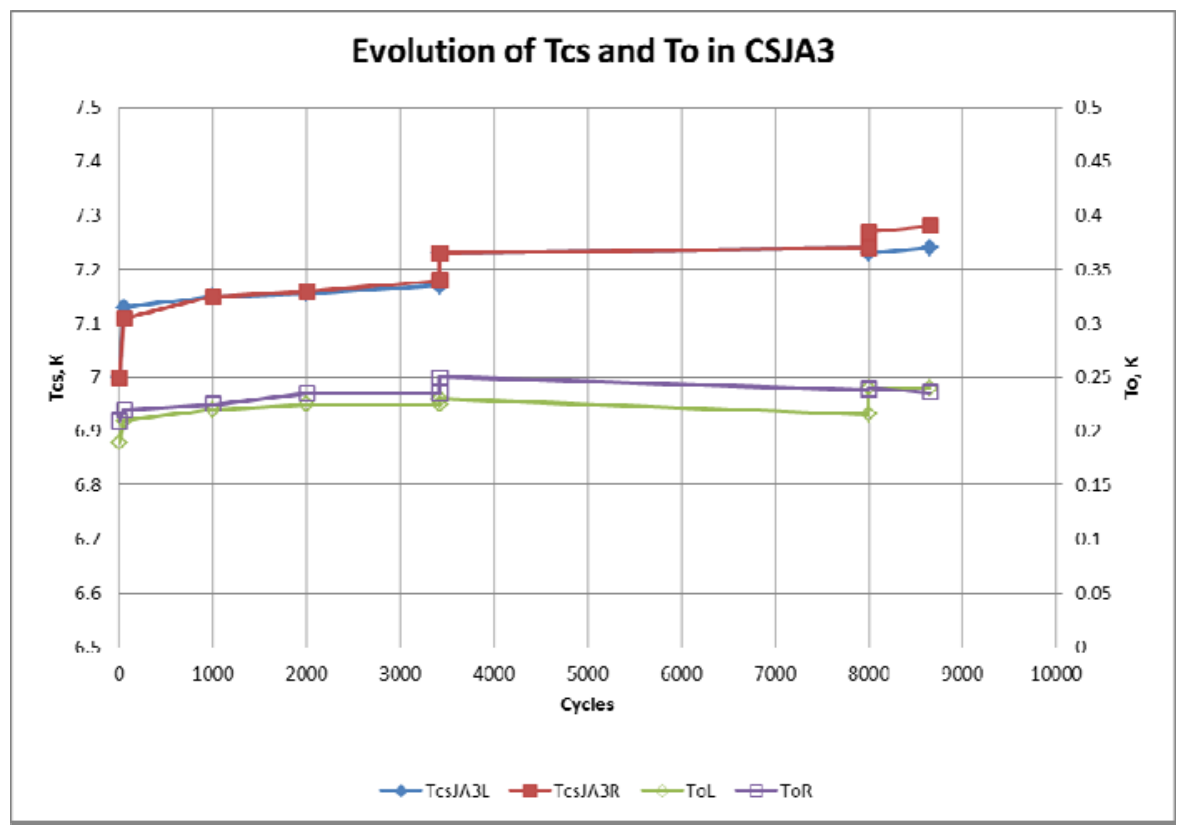

Fig. 7. Evolution of the Tcs and To parameters in CSJA3 [5] conductor.

\section{Speculation about Tcs growth nature in the selected CS conductors}

After reviewing different cases of Tcs and To behavior in CICC during cycling we can make the following speculations. It is reasonable to assume that at least two competing processes are occurring and the interplay can result in different patterns: filament fracture and strain redistribution. We include filament breaking and plastic deformation of the strand without filament fracture that causes similar effects under the name of filament fracture for convenience. In this case To increases and the transition becomes broader.

Re-distribution of the strain among strands in the CICC that can cause more or less favorable strain conditions than the original "virgin" condition. We observed a "relaxation" on the strand in the high field region of the TFUS1 sample [16] using penetrating voltage taps that are installed on the same strand of the cable.

When Tcs and To both degrade (in the sense Tcs decreases with cycles and To increases, as most of the CICC conductors) that falls into the category of events when the fracture of the filaments is taking place and strain re-distribution is either insignificant or overwhelmed. Even if Tcs resembles stabilization, but To continuing its growth, it indicates that the fracture of the filaments is still taking place and will manifest itself in the following cycling or after warm-upcooldown cycle.

If Tcs decreases slightly and To does not change significantly after short cycling history, like in USTF1 [16], it may mean that there is a some strain redistribution inside the cable that is not accompanied by the extended filament fracture. Obviously, this strain re-distribution is more favorable in the original condition than the one after the cycles.

Although the growth of Tcs in CICC is rare, it was observed in a few samples. For example, the TFUS3 conductors demonstrated a small growth, after which it seemed to saturate 
[17], both in terms of Tcs and To parameters. This case could also be interpreted that strain redistribution takes place under EM cycling, but the strain distribution is becoming more favorable than the original condition.

In the five CS conductors with the short twist pitches [4-6], the Tcs demonstrated amazingly similar growth but To parameters showed slight growth and saturation after sufficient amount of cycles. This condition demonstrates that the strands move under the EM cycling and the strain re-distribution brings slightly more favorable conditions for the strands than in the virgin conditions. The broadness change is insignificant, suggesting no fracture filaments damage.

\section{Summary}

Transition to the normal state is characterized by two parameters - the point when electrical field crosses "conventional" critical parameter (Tcs or Ic) and the broadness of transition. In this paper we show that the broadness of transition gives additional valuable information about character of degradation. The broadness of transition is often a more sensitive indication of degradation than the degrading parameter itself (Tcs or Ic). Growth of the broadness parameter (To) or reduction of $\mathrm{N}$-value has to stabilize before it can be concluded that degradation versus cycles has ended.

\section{Acknowledgement}

I am grateful to Wayne Reiersen (PPPL/US ITER) and to Wayne Meier (LLNL) for fruitful discussions and suggestions to the content of this paper. This manuscript has been authored by UT-Battelle LLC under Contract No. DE-AC05-00OR22725 with the US Department of Energy and under auspices of the U.S. DOE under Contract DE-AC52-07NA27344. The US Government retains and the publisher, by accepting the article for publication, acknowledges that the US Government retains a nonexclusive, paid-up, irrevocable, worldwide license to publish or reproduce the published form of this manuscript, or allow others to do so, for United States Government purposes.

\section{References}

1. N. Martovetsky, Full size TF CICC testing implications, Low Temperature Superconductors workshop at NHMFL, 2008, unpublished

2. N. Martovetsky, ITER CS conductor qualification progress, Low Temperature Superconductors workshop at Providence, RI, 2011,

3. A. Devred, private communications, November 2012

4. CSIO2 test results and parameters table, CRPP database and ITER IDM

5. CSJA3 test results and parameters table CRPP database and ITER IDM

6. CSJA5 test results, and parameters table, CRPP database and ITER IDM

7. G. L. Dorofeev, A. B. Imenitov, and E. Yu. Klimenko, Cryogenics 20, 307 (1980).

8. P. W. Anderson, Theory of Flux Creep in Hard Superconductors, Phys. Rev. Lett. 9, 309 (1962).

9. E. Yu. Klimenko, A. B. Imenitov, S. V. Shavkin, P. V. Volkov Resistance-current curves of high pinning superconductors Journal of Experimental and Theoretical Physics January 2005, Volume 100 , Issue 1 , pp 50-65

10. P. Bruzzone, B. Stepanov, ITER database, CRPP,SULTAN facility 
11. E.Yu. Klimenko, N. N. Martovetsky, Stability of the Superconducting Wires. Modern State of the Theory, IEEE Trans. on Magn.V.28 No.1, 1992, p.842-845

12. Anashkin I.O., Klimenko E.Yu., Lelekhov S.A., N. N. Martovetsky, S.I. Novikov et.al, Study of model coils made of a T-15 conductor, Atomic Energy, 1984, v.57, issue 6, p.401-404 (in Russian)

13. N. Martovetsky, Assessment of the CS Insert Tcs data: speculations on possible causes of initial degradation, memo 12/28/2000.

14. A. Godeke and H. Krooshoop et al, "Wide temperature and field scaling relations in Nb3Sn ITER strands”, University of Twente, Final report to NET, September 2000

15. N. Martovetsky, P. Michael, J. Minervini et al, "ITER CS Model Coil and CS Insert test results" IEEE Trans. Appl. Superconduct., vol. 11, N1, pp. 2030-2033, March 2001

16. N. Martovetsky, D. Hatfield, J.R. Miller, C-Y Gung, J.H. Schultz, N. Cheggour, L.F. Goodrich, P. Bruzzone, B. Stepanov, R. Wesche, B. Seeber, Test results of the first US ITER conductor in SULTAN, IEEE Trans. Appl. Superconduct., Volume 19, No 3, June 2009 Page(s):1478 - 1481

17. N.N. Martovetsky, D.R. Hatfield, JR Miller, P. Bruzzone, B. Stepanov, B. Seeber, Qualification of the US-made conductors for ITER toroidal field (TF) Magnet System, IEEE Trans. Appl.

Superconduct., vol. 20, No. 3, June 2010, p 466-469 